\title{
Complexity and synchronization
}

\author{
Malgorzata Turalska, ${ }^{1}$ Mirko Lukovic, ${ }^{2,3}$ Bruce J. West, ${ }^{4}$ and Paolo Grigolini ${ }^{1,2,3,5}$ \\ ${ }^{1}$ Center for Nonlinear Science, University of North Texas, P.O. Box 311427, Denton, Texas 76203-1427, USA \\ ${ }^{2}$ Dipartimento di Fisica “E. Fermi,” Università di Pisa, Largo Pontecorvo 3, 56127 Pisa, Italy \\ ${ }^{3}$ INFM, Largo Pontecorvo 3, 56127 Pisa, Italy \\ ${ }^{4}$ Mathematical and Information Science Directorate, US Army Research Office, Research Triangle Park, North Carolina 27709, USA \\ ${ }^{5}$ Istituto per i Processi Chimico-Fisici, Area della Ricerca del CNR, Via G. Moruzzi, 1-56124 Pisa, Italy
}

(Received 12 May 2009; published 14 August 2009)

\begin{abstract}
We study a fully connected network (cluster) of interacting two-state units as a model of cooperative decision making. Each unit in isolation generates a Poisson process with rate $g$. We show that when the number of nodes is finite, the decision-making process becomes intermittent. The decision-time distribution density is characterized by inverse power-law behavior with index $\mu=1.5$ and is exponentially truncated. We find that the condition of perfect consensus is recovered by means of a fat tail that becomes more and more extended with increasing number of nodes $N$. The intermittent dynamics of the global variable are described by the motion of a particle in a double well potential. The particle spends a portion of the total time $\tau_{S}$ at the top of the potential barrier. Using theoretical and numerical arguments it is proved that $\tau_{S} \propto(1 / g) \ln ($ const $\times N)$. The second portion of its time, $\tau_{K}$, is spent by the particle at the bottom of the potential well and it is given by $\tau_{K}$ $=(1 / g) \exp ($ const $\times N)$. We show that the time $\tau_{K}$ is responsible for the Kramers fat tail. This generates a stronger ergodicity breakdown than that generated by the inverse power law without truncation. We establish that the condition of partial consensus can be transmitted from one cluster to another provided that both networks are in a cooperative condition. No significant information transmission is possible if one of the two networks is not yet self-organized. We find that partitioning a large network into a set of smaller interacting clusters has the effect of converting the fat Kramers tail into an inverse power law with $\mu=1.5$.
\end{abstract}

DOI: 10.1103/PhysRevE.80.021110

PACS number(s): 05.40.-a, 02.50.Ey

\section{INTRODUCTION}

The phenomenon of decision making is by its nature interdisciplinary and its understanding has attracted an ever increasing number of investigators [1-3]. One aspect of the broad interest stems from the fact that group decisions are just as important to human beings as they are to other social animals [1], thereby bridging the gap between sociology and biology.

On the other hand, experiments on collective behavior in humans [2] show that the topology of the human group network can affect the speed of the decision-making process. This observation establishes a connection with the physics of living neural networks [4] where the transport of information along the network seems to play a crucial role for the network's function [5].

The decision-making process is consequently characterized by the joint action of two distinct forms of complexity: dynamic complexity and network complexity. The transport of information between complex networks occurs through the complexity matching (CM) phenomenon which may depend on the joint action of the two kinds of complexity. The remainder of Sec. I illustrates these three concepts.

\section{A. Structural complexity}

The pioneering paper by Watts and Strogatz [6] established that real networks depart from the condition of total randomness, which is characterized by weak clustering, and show surprisingly large clustering coefficients instead. Barabási and Albert [7] pointed out that real networks are also characterized by the scale-free condition, a property that implies an inverse power-law distribution density of links per node. Since then, attempts have been made to create a model that generates both high-clustering and scale-free properties. One such attempt is that of Holme and Kim [8], who assumed that the new nodes not only establish links preferentially with nodes already having a high number of links but they also establish additional links with the neighbors of these nodes. Another interesting proposal suggests that the networks grow by incorporating units that are already clustered, for instance, as triangles [9].

The structure of scale-free networks with high-clustering coefficients [10] consists of many clusters, each with a local leader or hub. There exists hub-to-hub coupling that can be interpreted as a channel of communication between clusters. This hub-to-hub connectivity suggests that real networks may rest on the presence of both scale-free and highclustering properties to realize maximum efficiency of the decision-making process.

Making general predictions on the influence of network complexity on the efficiency of a decision-making model is a hard problem due the fact that the efficiency of a model may be different from the topological or structural efficiency [11] and may depend on the specific nature of the model.

As a significant example of decision-making model dependence on network structural complexity we refer the reader to the recent work in Ref. [12], which shows that the scale-free networks are not necessarily those that reach collective decisions the fastest.

We are convinced that the decision-making model proposed herein may not produce faster decisions than if they were scale-free and may not benefit from the hierarchical structure of the network. We believe that decision making 
benefits from the existence of clusters, and for this reason, we limit our discussion of network structural complexity. In fact we restrict analysis to the very simple case of a limited number of interacting clusters and each cluster within the model is fully connected. On the other hand the network of clusters departs from the all-to-all coupling condition because only a limited number of elements from within one cluster are allowed to interact with another cluster.

\section{B. Dynamic complexity}

Dynamic complexity can be realized, in principle, by using a network whose structure is not complex, fitting the fully coupled condition within a cluster. Dynamic complexity is closely related to the phase-transition process occurring in physical systems, corresponding to the emergence of a global behavior, for instance, the synchronization of a fully coupled set of Kuramoto oscillators [13]. The single nodes used herein are not Kuramoto oscillators but are the stochastic oscillators recently studied by three research groups [14-16]. The main reason for our choice is that the two-state version of this model is that it provides a description of a simple decision-making process. The single units, in fact, correspond to individuals who have to choose between two possibilities, 1 and -1 . In the absence of cooperation, the individuals act independently; the choice is virtually equivalent to tossing a coin. Actually, an individual's decision is kept for a time on the order of $1 / \mathrm{g}$ after which time it might change. Cooperation within a cluster, modeled by the interactions of the two-state nodes, implies that during the decision-making process the dynamics of each unit is influenced by the choices made by the units to which each unit is linked. We show that an effect of cooperation is that the decision making can be made global and, due to selforganization, the entire network behaves as a single macroscopic unit. In the asymptotic case of all-to-all coupling in a dynamic network with an infinite number of units, the choice of either 1 or -1 becomes permanent.

As we prove in this paper, in the case of a finite number of nodes, the choice of either 1 or -1 does not last very long. The internode coupling generates intermittent behavior in the time series representing the overall state of the cluster. The non-Poisson character of the network time series is the most significant signature of dynamic complexity.

Investigators [16] used the two-state version of this model to provide a convenient picture of the fluorescent intermittency of blinking quantum dots. In fact, the waiting-time distribution of the "light on" and "light off" states is an inverse power law with index $\mu=1.5$ and the corresponding sequence is a renewal process [16], in accordance with the statistical analysis of real intermittent signals [17]. Bianco et al. [16] did not discuss the origin of the truncation of this inverse power law, interpreting it to be a property obscuring the true complexity underlying the emergence of the inverse power law. To prepare the ground for studying the transmission of information from one cluster to another, which is related, as we shall see, to network complexity, herein we explore the detailed mechanisms responsible for the truncation of the inverse power law. Moreover we prove that rather than being irrelevant, this unexpected and disturbing truncation of the inverse power law is an important part of the nonergodic nature of the process. Consequently, the truncation plays an important role in the transmission of information from one cluster to another.

In summary, dynamic complexity is signaled by the nonPoisson intermittence emerging in the condition of phase transition from uncoordinated short-time decisions to global decision-making process in which a cluster reaches near consensus. We shall refer to this latter condition as selforganization. In fact, as we shall see, the global decisionmaking process is realized by the internal interaction between the single units of the system rather than by an external control parameter, such as temperature in physical networks.

It is important to stress that to some extent the dynamic process of this paper is related to the issue of thermally activated processes and of dynamically bistable networks $[18,19]$. This fact is made evident herein where the decisionmaking process is described in part by a bistable potential that is illuminated with the help of Kramers theory [20]. However, the true source of fluctuation in these nonphysics networks is not thermal, thereby assigning to complex dynamics a nature different from that of ordinary phasetransition processes, and explains our adopting of the term self-organization.

\section{Complexity matching}

In the literature there are many papers devoted to the study of the global properties of complex networks, which are primarily concerned with network structure. The main focus of the present paper is on determining how the transfer of information from one cluster to another is facilitated by the self-organization within each of the clusters. A decision maker in isolation makes decisions lasting for finite times, and the sequence of time durations of these decisions is a renewal Poisson process. A self-organized cluster retains a decision for a much longer time than that of a decision maker in isolation. However, the time duration of a decision made by a self-organized cluster is also finite and the selforganized cluster also generates a renewal time series: the main difference between the self-organized and isolated decision maker is that the time series generated by the selforganized cluster is not a Poisson process. The abrupt decision changes in a cluster are crucial events [16]. In accordance with existing nomenclature [16] we denote as crucial the renewal events when the time distance between two consecutive events is a stochastic quantity whose mean value is infinite. We denote with the symbol $\psi(\tau)$ the distribution density of decision-time durations $\tau$. In the case where $\psi(\tau) \propto 1 / \tau^{\mu}$, the renewal events are crucial if $\mu<2$. Herein the idea of an event being crucial is realized by fat tails that in the finite time region accessibility to information turns out to be slower than in the inverse power-law tails. Thus the response of a self-organized cluster to an external stimulus is expected to be characterized by the CM principle [21]. One consequence of the CM principle is that a selforganized cluster is only sensitive to the influence of another 
self-organized cluster. The upshot of analysis and computations made herein is the assessment of how CM dominates the communication between network clusters. It is expected that this discovery can be used to establish guidelines for the study of the decision-making process of sets of decision makers that are the members of a complex network. In conclusion, we think that the $\mathrm{CM}$ effect, discussed in this paper, may help us to establish the connection between dynamic and network complexity.

The outline of this paper is as follows. In Sec. II we present a concise review of the dynamic two-state model [16] applied to decision making so that the present paper is as self-contained as possible. In Sec. III we explain why the decision-making survival probability has the typical form of an inverse power law that is truncated by an exponential shoulder. To prove that the decision-making process emerging from the cooperation of many units is renewal we devote Sec. IV to illustrating the results of numerical experiments that assesses the renewal nature of this process. In Sec. V we show the weak breakdown of ergodicity with effects that are very similar to those currently assessed by the observation of inverse power-law processes, with no tail truncation. Section VI illustrates how the transfer of information between two networks depends on their relative complexity. In Sec. VII we illustrate the effect of splitting the networks into four weakly coupled parts. Finally, we devote Sec. VIII to concluding remarks and plans for future work.

\section{THEORETICAL PREDICTION IN THE CASE OF ALL- TO-ALL COUPLING AND $N=\infty$}

This section is devoted to a concise review of the decision-making model [16]. This will allow us to define the barrier intensity $Q$, which is similar to the energy barrier between the two wells of a double well potential used by Kramers [20]. The parameter $Q$ is useful for the theoretical interpretation of the numerical results presented subsequently.

\section{A. Cooperative decision-making model}

The dynamic of a single unit is described by the two-state master equation,

$$
\begin{aligned}
& \frac{d}{d t} p_{1}=-g_{12} p_{1}+g_{21} p_{2}, \\
& \frac{d}{d t} p_{2}=-g_{21} p_{2}+g_{12} p_{1},
\end{aligned}
$$

where $p_{j}$ is the probability of being in the state $|j\rangle$ and $p_{1}$ $+p_{2}=1$. The states $|1\rangle$ and $|2\rangle$ correspond to the values 1 and -1 , respectively.

When there is no network the single unit is isolated and the transition rates are $g_{12}=g_{21}=g \ll 1$. From a formal point of view the master equation can be derived by assuming that the decision between the two alternatives is made by this node tossing a fair coin and then retaining this decision for a time $\tau$ derived from the exponential distribution density,

$$
\psi(\tau)=r \exp (-r \tau),
$$

with the transition rate given by $g=r / 2$. Here $r$ is the rate at which decisions are made or a number of decisions made per unit time. In this simple case once a decision is made, it is kept for an average time $\tau_{D}$ given by the inverse of the rate,

$$
\tau_{D}=\frac{1}{r}=\frac{1}{2 g} .
$$

Each node of a given network is occupied by a dynamic unit described by the master equation of Eqs. (1) and (2). A given unit is perturbed by all the other units to which it is directly connected according to the prescription,

$$
g_{i j}(t)=g \exp \left\{K\left[\pi_{j}(t)-\pi_{i}(t)\right]\right\} \delta_{j, i \pm 1},
$$

where

$$
\pi_{s}=\frac{M_{s}}{M} .
$$

$M$ denotes the number of nodes connected to the site that we are considering and $M_{s}$ is the number of the nearest-neighbor nodes that are in the state $s, s=1,2$. The parameter $K$ is the control parameter, generating the coupling factor

$$
C_{i, j}=\exp \left\{K\left[\pi_{j}(t)-\pi_{i}(t)\right]\right\} .
$$

The single unit now keeps its decision for a time longer or shorter than $\tau_{D}$ according to the choices made by its neighbors. If half of its neighbors are in the state $|j\rangle$ and half in the state $|i\rangle$, the single unit remains in the state $|i\rangle$ as it would in isolation. If the majority of its neighbors are already in the state $|j\rangle$, the single unit makes its decision of selecting $|j\rangle$ earlier than in isolation. In the opposite state when the majority of its neighbors are still in the state $|i\rangle$ the single unit keeps the original decision for a longer time.

The key point is that the master equation of each site is well defined, but it is a fluctuating master equation. In fact the transition coefficients $g_{i j}$ depend on the quantities $\pi_{s}$, which have random values depending on the stochastic time evolution of the environment of a specific site. As a matter of fact, we may define another frequency,

$$
\Sigma_{s}=\frac{J_{s}}{J},
$$

where $J$ denotes the total number of sites in the network. It is evident that the quantity $\Sigma_{s}$ is also an erratic function of time even if it is expected to be smoother than $\pi_{s}$. In fact, $\Sigma_{s}$ is a global property, obtained from the observation of the entire network, while $\pi_{s}$ is a property of the environment of a given site. The smaller the cluster, the more erratic the quantity $\pi_{s}$. We also define the stochastic variable

$$
\xi(t)=\Sigma_{1}(t)-\Sigma_{2}(t),
$$

whose variability is characteristic of the network.

\section{B. Stable condition}

Let us consider the all-to-all coupling case and assume that the total number of nodes, $N$, becomes infinite. In this case $\pi_{s}=\Sigma_{s}=p_{s}$, thereby yielding 


$$
g_{12}=g \exp \left[-K\left(p_{1}-p_{2}\right)\right]
$$

and

$$
g_{21}=g \exp \left[-K\left(p_{2}-p_{1}\right)\right]
$$

Let us define the difference in the probabilities

$$
\Pi \equiv p_{1}-p_{2} .
$$

Subtracting Eq. (2) from Eq. (1) yields the rate equation for the difference variable,

$$
\frac{d}{d t} \Pi=-\left(g_{12}+g_{21}\right) \Pi+\left(g_{21}-g_{12}\right) .
$$

By inserting Eqs. (10) and (11) into Eq. (13) we obtain

$$
\frac{d}{d t} \Pi=-g\left(e^{K \Pi}+e^{-K \Pi}\right) \Pi+g\left(e^{K \Pi}-e^{-K \Pi}\right)=-\frac{d V}{d \Pi} .
$$

For $K<1$, half of the nodes are in the state $|1\rangle$ and half are in the state $|2\rangle$. At the critical value $K=K_{c}=1$ a bifurcation occurs and the potential develops two wells separated by a barrier [Fig. 1(a)]. It is straightforward to prove that for $K$ $>1$, but very close to 1 , the barrier intensity $Q$ is given by

$$
Q=g\left(\frac{K-1}{K}\right)^{2}
$$

and the two equilibrium values of $\Pi$ are

$$
\Pi_{e q}= \pm\left(\frac{2(K-1)}{K}\right)^{1 / 2} \text {. }
$$

Figures 1(b) and 1(c) serve to illustrate the agreement between the barrier intensity $Q$ and the equilibrium values $\Pi_{e q}$ and their approximations by Eqs. (15) and (16), respectively.

At $K$ slightly larger than the critical value $K_{c}=1$, a majority decision emerges. The condition of perfect consensus is reached for $K \rightarrow \infty$. In this case $\Pi$ gets either the value of 1 or of -1 . It is important to stress that, even if for $K>1$ but finite the consensus is only partial, the network is in an equilibrium condition, where the majority and the minority keep their choice forever, with no change of opinion.

\section{Fluctuating condition}

The condition for the existence of the stable condition can be violated. There are two main causes for this violation. The first is that the number of nodes is finite. The second cause is the lack of the all-to-all coupling. In real networks both sources of equilibrium breakdown are expected to occur. Herein we consider all-to-all networks, with $N$ nodes, where the variable $\xi$ becomes stochastic and its time evolution is described by the Langevin equation,

$$
\frac{d \xi(t)}{d t}=-\frac{d V(\xi)}{d \xi}+\eta(t)
$$

where $\eta(t)$ is a random fluctuation whose intensity is proportional to $1 / \sqrt{N}$. Although Eq. (17) is written in the continuous time representation, in practice the numerical calcula-
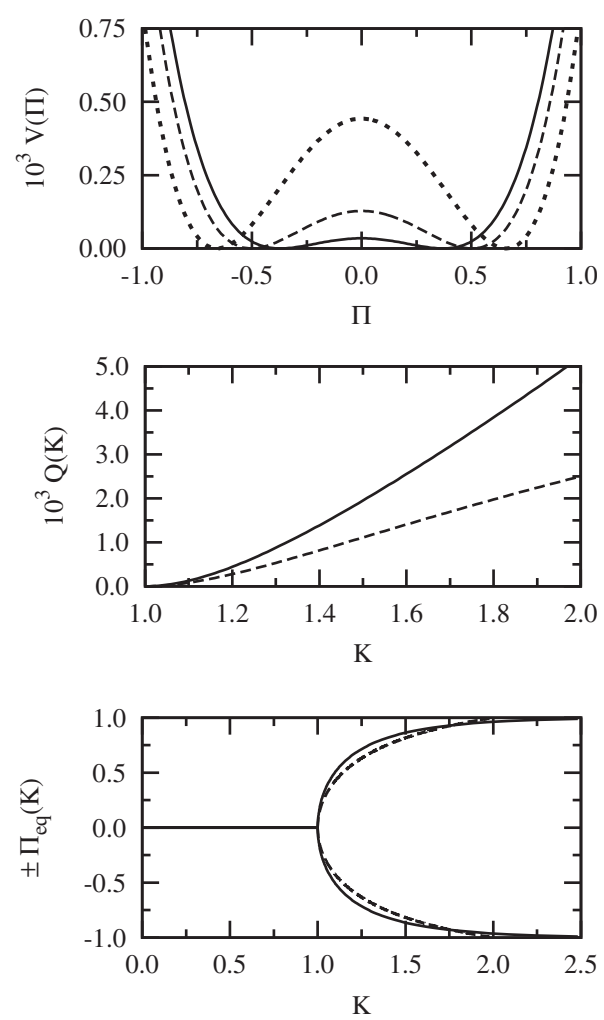

FIG. 1. (Top) The double well potential $V(\Pi)$ as a function of $\Pi$ for $g=0.01$ and different values of the coupling constant $K$. Solid line for $K=1.05$, dashed line for $K=1.10$, and dotted line for $K$ =1.20. (Middle) The barrier intensity $Q(K)$ as a function of the coupling constant $K$ for $g=0.01$. Solid line for the barrier intensity derived from Eq. (14); dashed line for barrier intensity approximated by Eq. (15). (Bottom) The equilibrium value $\Pi_{e q}(K)$ as a function of the coupling constant $K$ for $g=0.01$. Solid line for values derived from Eq. (14); dashed line for values approximated by Eq. (16).

tions of this paper correspond to the adoption of a finite integration time step $\Delta t=1$. Note that the stochastic rate equation [Eq. (17)] replaces the master equation [Eq. (14)] in the case of a finite $N$ and that Eq. (14) is recovered in the ideal case $N=\infty$. We also consider the case of two or more all-to-all coupled networks that mutually interact by means of only a fraction of all possible links. In this case the resulting network departs from the picture of Eq. (17). In fact in this latter case, we can no longer invoke the assumption that $\pi_{s}(t)$ is independent of the node considered. We may describe, for instance, the coupling between two all-to-all networks as corresponding to the interaction between two Langevin equations of the same kind as Eq. (17) with stochastic forces of different intensities if the two clusters have a different number of nodes.

\section{CASE OF A FINITE NUMBER OF NODES: BEYOND THE KRAMERS-SUZUKI THEORY}

In this section we consider the all-to-all coupling condition, with a finite number of nodes, $N$. In Fig. 2 the fluctuating variable $\xi(t)$ is depicted as a function of time under 

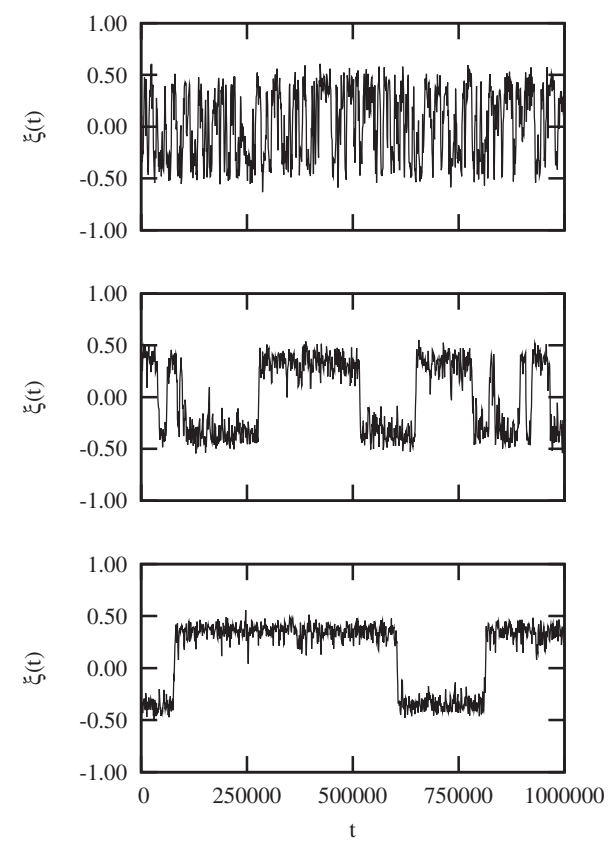

FIG. 2. The fluctuation of the mean-field-average phase as a function of time. (Top) For a system of $N=500$ clocks, $K=1.05$, and $g=0.01$. (Middle) For a system of $N=1500$ clocks, $K=1.05$, and $g=0.01$. (Bottom) For a system of $N=2500$ clocks, $K=1.05$, and $g=0.01$.

differing conditions. Notice that with increasing $N$ the fluctuation $\xi(t)$ becomes more distinctly dichotomous, with an increasingly sharp transition from the "up" to the "down" state. This pattern corresponds to the entire network keeping a decision for a longer and longer time as the size of the network increases. The ideal condition of a decision lasting forever is reached in the ideal case $N=\infty$.

We define as an event the abrupt transition from the up (down) to the down (up) state. We also notice that there exist transitions from $\xi(t)>0[\xi(t)<0]$ to $\xi(t)<0[\xi(t)>0]$ with a virtually vanishing residence time in the state up (down) on the time scale of these figures. These decisions of very shorttime duration are important for the exchange of information between clusters and cannot be ignored. They are responsible for the inverse power-law regime of the distribution density $\psi(\tau)$. We decided to record both long and short-time durations of the global choices, and for this reason we define as an event the crossing of the origin of the $\xi$ axis. The time duration of a choice is then the time interval between two consecutive recrossings of the origin of the $\xi$ axis.

In Fig. 3 we plot the survival probability

$$
\Psi(t)=\int_{t}^{\infty} d \tau \psi(\tau) .
$$

For values of $N$ of the order of $N \approx 10$ the survival probability is similar to that of a single unit in the isolation condition,

$$
\Psi(t)=\exp (-g t) .
$$

For values of $N$ on the order of 100 the time region of the Poisson process of Eq. (19) is turned into an inverse power law with index $\mu-1 \approx 0.5$. A further increase in $N$ does not

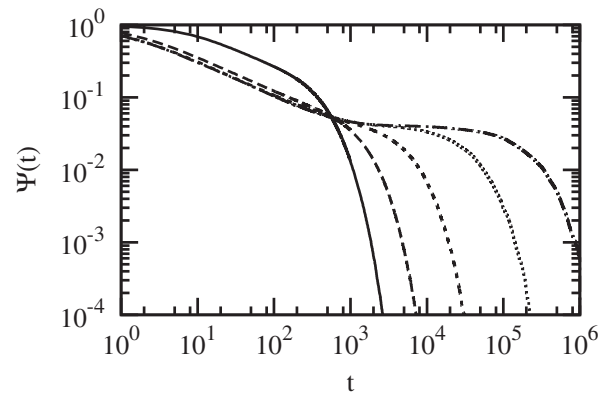

FIG. 3. Survival probability $\Psi(t)$ function for $K=1.05, g=0.01$, and increasing network size $N$. Thin solid line for a system of $N=10$ clocks, long dashed line for $N=100$, short dashed line for $N=500$, dotted line for $N=1500$, and dashed dotted line for $N=2500$.

affect the time region defined approximately by $t<1 / g$, and it has only the effect of producing a more and more extended shoulder that we refer to as the Kramers shoulder due to the theoretical interpretation of its cause.

We note that the process becomes ergodic for times larger than the Kramers time $\tau_{K} \approx \exp ($ const $\times Q N)$ (we subsequently explain the physical origin of this time). The much shorter time $1 / \mathrm{g}$ is close to the Suzuki time $\tau_{S}$, which is also explained later in this section.

Imagine producing infinitely many realizations of the decision-making process all with the same initial condition $\xi=0$. The probability distribution density $P(\xi, t)$ will evolve from a Dirac delta function $\delta(\xi)$ to the final equilibrium density,

$$
P_{e q}(\xi) \propto \exp \left(-\frac{V(\xi)}{D}\right) .
$$

The parameter $D$ is the diffusion coefficient determined by the strength of the noise $\eta(t)$ in Eq. (17). We set

$$
D=\frac{g}{N} .
$$

The equilibrium distribution [Eq. (20)] is realized in the Kramers time scale $t \sim \tau_{K}$. In this time scale the decisionmaking process is expected to be ergodic. However, we have to take into account that our observation process rests on sequences, whose size $L$ ranges from $L=10^{4}$ to $L=10^{7}$. Note that $N \approx 100$ already generates a Kramers time on the order of $\tau_{K}=10^{4}$. As a consequence, we reach the preliminary conclusion that the observation process limited to the range of $L$ forces us to interpret the decision-making process as being out of equilibrium if $\tau_{K}$ is of the order of $L$.

To make theoretical predictions on the decision-time distribution density $\psi(\tau)$ we use the formula [22]

$$
P(\xi=0, t) \propto R(t)=\sum_{n=1}^{\infty} \psi_{n}(t),
$$

where $\psi_{n}(t)$ denotes the probability density for the network to return to the origin for the $n$th time, given the condition that it was at the origin at $t=0$. The quantity $R(t)$ is the number of returns to the origin per unit of time, given the 
condition that the network was at the origin at time $t=0$. The physical justification of this formula is that in the microscopic time scale the network leaves the origin at the first time step due to the fluctuation $\eta$.

Using the renewal condition we connect $\psi_{n}(t)$ to $\psi_{n-1}(t)$ by means of

$$
\psi_{n}(t)=\int_{0}^{t} d t^{\prime} \psi_{n}\left(t-t^{\prime}\right) \psi_{1}\left(t^{\prime}\right),
$$

with $\psi_{1}(t)=\psi(t)$, the decision-time distribution density. Let us use the method of Laplace transform and the notation $\hat{f}(u) \equiv \int_{0}^{\infty} d t \exp (-u t) f(t)$. It is straightforward to prove that

$$
\hat{R}(u)=\frac{\hat{\psi}(u)}{1-\hat{\psi}(u)} .
$$

We note that $R(t)$ has the dimension of the inverse of time. Therefore it is convenient to write

$$
R(t)=k P(\xi=0, t),
$$

where $k$ is a convenient factor ensuring that both terms of Eq. (25) have the same physical dimensions. The choice of $k$ depends on whether we consider the short- or the long-time region. Let us assume we can completely neglect the fact that the network is regressing toward the equilibrium condition [Eq. (20)] or, more precisely, that the network is in a freediffusion situation corresponding to $V(\xi)=$ const. In this case the function $P(\xi=0, t)$ decays as $1 / t^{\delta}$, as prescribed by the rescaling property of a diffusion process with $\xi \propto t^{\delta}$. Thus we are led to

$$
R(t) \approx \frac{1}{\tau_{c}}\left(\frac{\tau_{c}}{t}\right)^{\delta},
$$

where $\tau_{c}$ is the time necessary to move from the discrete time evolution to a scaling regime, where $\delta$ can be clearly evaluated. We estimate $\tau_{c}$ to be about ten time larger than the integration time step $\Delta t$ that, as earlier stated, is set equal to 1 in the numerical calculations of this paper. Consequently, $\tau_{c} \approx 10$. The regression to the origin is virtually independent of $D$, which controls the intensity of the fluctuations but not the regression times. A trajectory leaving the axis $\xi=0$ at $t_{i}$ and coming back to it at $t_{i+1}$, in between these two times may depart from the origin by quantities whose modulus is proportional to $D$, but the time interval $\left|t_{i+1}-t_{i}\right|$ is determined by the scaling coefficient $\delta$. Using the Tauberian theorem,

$$
\frac{1}{t^{\delta}} \rightarrow \frac{\Gamma(1-\delta)}{u^{1-\delta}},
$$

we convert Eq. (26) into

$$
\hat{R}(u) \approx\left(\tau_{c} u\right)^{(\delta-1)} \Gamma(1-\delta) .
$$

To go from $R(t)$ to $\psi(t)$ we use Eq. (24) that yields

$$
\hat{\psi}(u)=1-\frac{1}{\hat{R}(u)} \approx 1-\frac{\left(\tau_{c} u\right)^{(1-\delta)}}{\Gamma(1-\delta)} .
$$

Let us assume that

$$
\psi(t)=(\mu-1) \frac{T^{\mu-1}}{(t+T)^{\mu}} .
$$

This distribution density is properly normalized and affords a simple way to ensure the asymptotic time property $\psi(t) \propto \frac{1}{t^{\mu}}$ with no divergence at $t=0$. The parameter $T$ determines the size of the time interval within which the distribution density $\psi(t)$ is not yet an inverse power law. The Laplace transform of $\psi(t)$ is given by

$$
\hat{\psi}(u) \approx 1-\Gamma(2-\mu)(u T)^{\mu-1} .
$$

By comparing Eq. (31) to Eq. (29) we get

$$
\mu=2-\delta
$$

and

$$
T=\frac{\tau_{c}}{[\Gamma(1-\delta) \Gamma(\delta)]^{1 /(1-\delta)}} .
$$

We note that the diffusion process generated by the random fluctuation $\eta$ is ordinary, thereby yielding $\delta=0.5$ for the scaling of the left-hand side of Eq. (22) and, through Eq. (32),

$$
\mu=1.5 \text {. }
$$

Using Eq. (33) and $\delta=0.5$ we predict that

$$
T=\frac{\tau_{c}}{\Gamma(0.5)^{4}} \approx 0.1 \tau_{c} .
$$

Thus we estimate $T$ to be of the order of 1 , in accordance with the numerical results of Fig. 3.

How extended is this out-of-equilibrium process with an inverse power-law structure? To answer this important question we use the Suzuki theory [23] of 30 years ago. Suzuki established the time scale necessary to produce macroscopic order in a physicochemical system on the basis of weak fluctuations. This time is essentially the time spent by the network at the top of the barrier separating the two potential wells [see Fig. 1(a)].

When the diffusion trajectory $x(t)=\int_{0}^{t} d t^{\prime} \xi\left(t^{\prime}\right)$ departs sufficiently from the origin, it is attracted by the well bottoms and reaches one of them through fast deterministic motion. Subsequently, the network fluctuates around the well bottom until it eventually returns to the top of the barrier. This more extended time scale is determined by Kramers theory [20]. The Suzuki time scale [23] is

$$
\tau_{S} \approx \frac{1}{g} \ln (1 / D)
$$

This prediction assumes that the second derivative of the potential $V(\Pi)$ at the top of the barrier and at the bottoms of the two potential wells is of the order of 1 . The Suzuki time scale tends to increase upon increase in $N(1 / D \propto N)$ and this produces a time scale that has a weak logarithmic dependence on $N$ that can be ignored.

Now, let us move from the idealized condition that the network lives forever in the free-diffusion out-of-equilibrium regime to the opposite extreme of perfect thermodynamic 


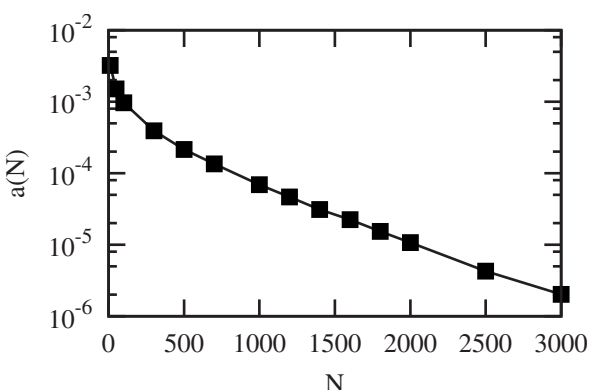

FIG. 4. The result of fitting Kramer's shoulder of the survival probability $\Psi(t)$ with an exponential function.

equilibrium. Imagine the capability (that we do not have) of observing the network on a time scale sufficiently large as to perceive the network in the equilibrium condition [Eq. (20)]. In this idealized condition Eq. (22) can be written as the time-independent constant,

$$
a=\sum_{n=1}^{\infty} \psi_{n}(t),
$$

where $a$ is the constant rate of the origin recrossings, which yields

$$
a=A \exp \left(-\frac{Q}{D}\right),
$$

where $Q$ and $D$ is given by Eqs. (15) and (21), respectively, and $A$ is a constant factor. In this case $\hat{R}(u)=a / u$ and Eq. (24) yields

$$
\psi(t)=a \exp (-a t) .
$$

In Fig. 4 we show the result of a fitting procedure of the Kramers shoulder that allows us to establish the excellent accuracy of the theoretical prediction of Eq. (38) and to define what is meant by the Kramers time scale $\tau_{K}$. The Kramers time scale is defined by

$$
\tau_{K}=1 / a .
$$

It is now evident that, as stated earlier, the Kramers time scale has an exponential dependence on $N\left[\tau_{K} \propto \exp (\right.$ const $\times N)]$.

\section{AGING EXPERIMENT}

This section is devoted to establishing that the sequence of opinion changes is renewal. The renewal property is essential to classifying the change in opinion events as crucial. The first condition, $\langle\tau\rangle=\infty$, has been established in Sec. III. We know that for $N$ larger than about 100 the mean time duration of a choice exceeds the maximum length of the sequence that we can observe and therefore is operationally infinite. This $N$ dependence is shown to generate ergodicity breakdown, but we have to establish that this breakdown occurs under the specific form of renewal ergodicity breakdown. We notice that the theoretical interpretation adopted in Sec. III may give the misleading impression that the process

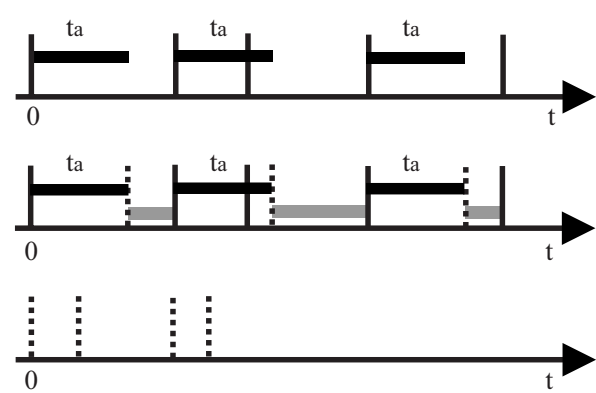

FIG. 5. Illustration of the aging experiment. Vertical bars on the top figure indicate the crucial events. Horizontal bar represents aging time $t_{a}$. Gray horizontal bars on the middle figure reflects waiting times for the aged system, which are represented as a sequence on time scale on bottom figure.

is not renewal and that first we have a sequence of short time corresponding to the inverse power-law distribution density with $\mu=1.5$ and later to a sequence of Kramers times. This is not so. If we locate the network on the origin $\xi=0$ at $t=0$, the network moves according to the diffusion-free arguments. If the sojourn time is shorter than the Suzuki time, it certainly belongs to this short-time distribution. However, since this short-time distribution is renewal, after a sojourn time smaller than $\tau_{S}$ we may have a new sojourn time that is much larger than $\tau_{S}$. This would certainly occur in the absence of the Kramers barrier and in the absence of the repulsion walls as well. The presence of the constraining walls converts this extended time into a much shorter one and, in the presence of the potential barrier, into the time of Kramers theory.

Although the renewal nature of the decision-making model has already been studied and assessed in the earlier work in Ref. [16], we notice that those preliminary results had to do with cases of a Kramers shoulder that was not very well pronounced. For this reason, we decided to re-examine the renewal property of this model. At the same time, we judged it important to give more details on the nature of the aging experiment used to establish the renewal nature of the process.

The aging experiment is a method of analysis of time series originally proposed by Allegrini et al. in Ref. [24]. As indicated by the sketch of Fig. 5, the aging experiment proceeds as follows. We adopt a window of size $t_{a}$, corresponding to the age of the network that we want to examine. We locate the left end of the window on the time of occurrence on an event, and we record the time interval between the right end of the window and the first event after emerging out of the window. It is evident that the adoption of windows of vanishing size corresponds to generating ordinary histograms. The histograms generated by $t_{a}>0$ produce different decision-time distribution densities, and these distribution densities, properly normalized, generate survival probabilities whose relaxation can be distinctly different from that of the ordinary survival probability. A nonergodic renewal process is expected to generate a relaxation that becomes slower and slower as $t_{a}$ increases. To establish whether the aging is renewal or not, we shuffle the sequence $\tau_{1}, \tau_{2}, \ldots, \tau_{n}, \ldots$ so as to realize a new time series that is renewal. We then apply the aging experiment to the shuffled sequence, and if the two 


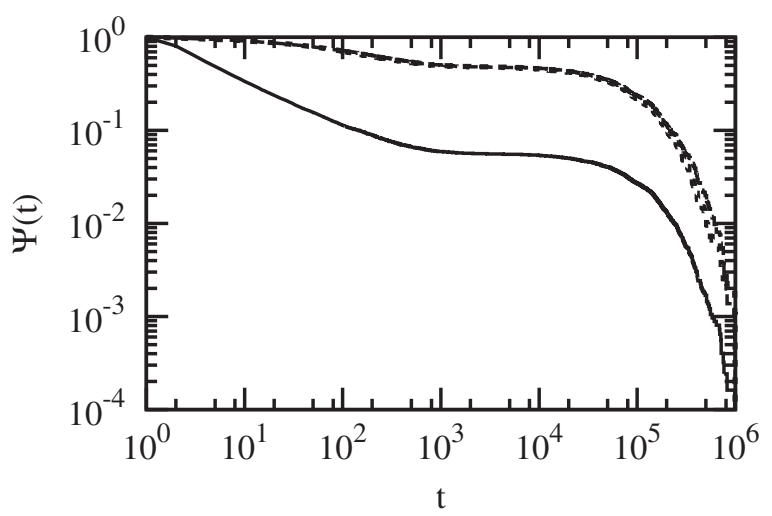

FIG. 6. Testing for renewal property. Survival probability function $\Psi(t)$ for $K=1.08, g=0.01$, and network size $N=1000$ (solid line). Survival probability $\Psi\left(t_{a}\right)$ aged by time $t_{a}=200$ (dashed line). Survival probability in the renewal case $\Psi_{r}\left(t_{a}\right)$ (dotted line).

survival probabilities coincide, we conclude that the process is renewal. The results of Fig. 6 show that the decisionmaking process is renewal with very good accuracy even though the survival probability has a very pronounced Kramers shoulder.

On the other hand to double check the renewal nature of the process we made an additional numerical experiment. We have generated a very large number of single realizations, 1000 , with $L=10^{7}$. This very long-time interval has been divided into three subintervals. The first subinterval runs from time $t=0$ to time $L_{1}=10^{4}$. The second runs from $L_{1}$ to $L-L_{1}$ and finally the last runs from $L-L_{1}$ to $L$. We make our observation on the first and the last subintervals, each of which has the length $L_{1}=10^{4}$. This time interval is too short to afford good statistics with only one realization and is the reason why we have made 1000 realizations. The results are illustrated in Fig. 7.

We see from Fig. 7 that there is no significant difference between the observations made after a time interval on the order of $10^{7}$ from one another. We also notice another interesting effect. The inverse power-law region, with a slightly larger power-law index $\mu=1.6$ rather than $\mu=1.5$, is extended over a larger number of decades thereby going beyond the Suzuki time limit.

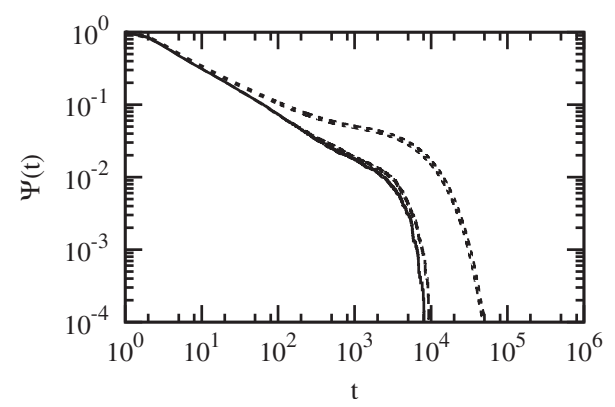

FIG. 7. Average over an ensemble of independent single realizations confirms renewal property of the process under investigation. Solid line for the survival probability function $\Psi(t)$ obtained for the first interval from time $t=0$ to time $L_{1}=10^{4}$. Dashed line for the survival probability function $\Psi(t)$ obtained for the last interval from time $L-L_{1}$ to $L$. Dotted line for the survival probability function $\Psi(t)$ obtained for the interval from time $t=0$ to time $L_{1}=10^{5}$.

\section{WEAK BREAKDOWN OF ERGODICITY}

In the literature ergodicity breakdown $[25,26]$ is associated with the emergence of an inverse power-law distribution density with $\mu<2$. Poisson processes are generally considered to be ergodic processes. This vision does not take into account the important fact that ergodicity or the breakdown of ergodicity depends on the time scale. If $\mu<2$, the mean time $\langle\tau\rangle$ diverges, and the process is not ergodic regardless the time scale adopted to prove the ergodicity breakdown. An illuminating example of this time scale dependence of ergodicity breakdown is given by the physics of blinking quantum dots, whose nonergodic nature is well assessed [27-29]. However, it is also known [30] (see also [31]) that the inverse power-law distribution density of light on and light off times has an upper (as well as a lower) bound. In our case the upper bound is not given by the Suzuki time, which is of the order of $\tau_{S}$, but it is given by the Kramers time [Eq. (40)].

Here we adopt the numerical experiments proposed by the authors in Refs. $[25,26]$ to establish the ergodicity breakdown for time scales shorter than $\tau_{K}$. We generate single realizations of the fluctuation $\xi$ with different values of $N$, so as to realize different values of $\tau_{K}$. We turn these sequences into symbolic sequences with the value of 1 when $\xi>0$ and the value of -1 when $\xi<0$. We consider a window of size $l$ and we move it along each symbolic sequence. For each window position there is a given number of + 's, $N_{+}$, and a given number of -'s, $N_{-}$, thereby generating the ratio

$$
z \equiv \frac{N_{+}-N_{-}}{N_{+}+N_{-}}
$$

whose range is $-1 \leq z \leq 1$. In the ergodic case the probability distribution density of $z$ is bell shaped centered at $z=0$. In the nonergodic case the distribution takes on the typical $U$ shape first identified by Lévy and Lamperti $[25,26]$. In Fig. 8 we see that for a fixed value of $l=20000$ the probability density function of $z$ changes from the $U$ form when $N=1000$ to a bell shape when $N=100$. It is evident that decreasing the value of $N$ has the effect of weakening the strong ergodicity breakdown. However, we notice that full ergodicity is realized when $p(z)$ is a Dirac delta function centered at $z=0$. The case $N=100$ is still very far away from the ergodic condition, in line with our observation that full ergodicity is realized with $N$ smaller than 100 . We therefore conclude that the process we are examining from $N=100$ to higher values is not ergodic.

\section{TRANSMISSION OF INFORMATION BETWEEN TWO NETWORKS}

We have established that our proposed model for cooperative decision making is a nonergodic renewal process. As a consequence, on the basis of the general arguments illustrated in the recent review [21], we expect that one selforganized cluster is insensitive to external perturbations not sharing its complexity. The easiest way to create an external perturbation with the same complexity of a given selforganized cluster $S$ is to generate a cluster $P$ identical to cluster $S$. The condition of weak perturbation is realized by 

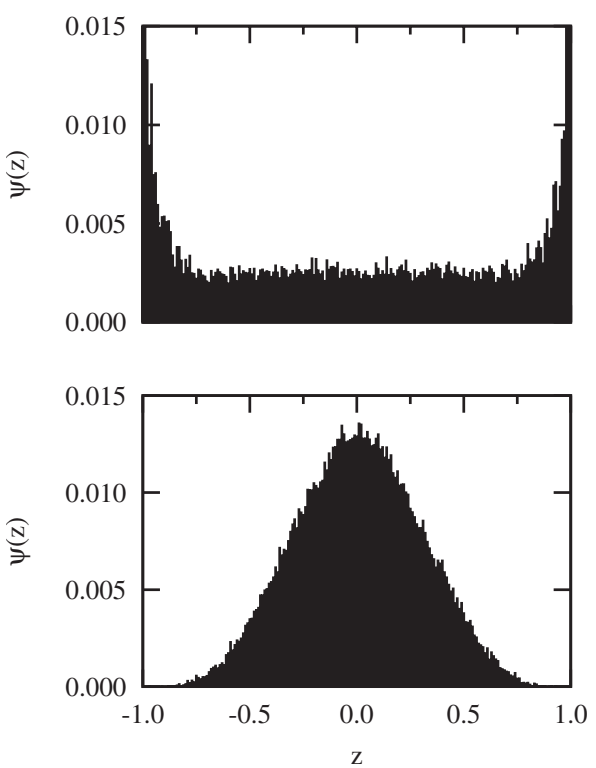

FIG. 8. (Top) Ergocity test performed for the fluctuation $\xi(t)$ obtained for a network with parameters $K=1.05, g=0.01$, and $N=1000$. (Bottom) Ergocity test performed for the fluctuation $\xi(t)$ obtained for a network with parameters $K=1.05, g=0.01$, and $N=100$. All tests were performed with window of length 20000 .

assuming that a small fraction $F$ of the $N$ nodes of $S$ is linked to all the nodes of $P$. To ensure the nature of an external perturbation with dynamics independent of $S$ we set the condition that the $N$ nodes of $P$ are not affected by the $F N$ nodes of $S$ that they perturb. To make it possible to realize a condition far from the $\mathrm{CM}$ condition, we do not set the rigid constraint $K_{P}=K_{S}$. Additionally, we define perturbation coupling constant $K_{P S}$, which is used to realize the change in the transition rate $g_{i j}$ for the fraction $F N$ of nodes of network $S$ due to the coupling with network $P$.

In Fig. 9 we show a variety of cases: (a) both clusters $S$ and $P$ are self-organized but not interacting; (b) both clusters $S$ and $P$ are self-organized and weakly interacting; (c) cluster $P$ is self-organized and $S$ is not, but $P$ perturbs $S$; and finally (d) cluster $S$ is self-organized and $P$ is not, but $P$ perturbs $S$. It is evident that in most cases there is no synchronization. The comparison of Figs. 9(a) and 9(b) shows that the coupling between two self-organized clusters results in synchronization when $K_{S} \leq K_{P}$. In the case presented on Fig. 9(a) there exists a mismatch between lengths of consecutive laminar regions. As marked on the plot, one can distinguish regions where the perturbation $P$ is positive and the fluctuation of the mean-field of network $S$ is negative in the first portion and positive in the second portion of the region. This is made possible by the fact that $K_{S}<K_{P}$. Consequently, the $S$ laminar regions are less extended than the $P$ laminar regions. Figure 9(b) shows that in spite of this mismatch in the uncoupled case, when the coupling is switched on, a very good synchronization emerges: the weak perturbation is sufficient to extend the length of the laminar regions of $S$.

Therefore, only when both clusters are self-organized there is synchronization through the cluster to cluster interaction. To establish all this on a more quantitative level we evaluate the asymptotic properties of the cross-correlation function,
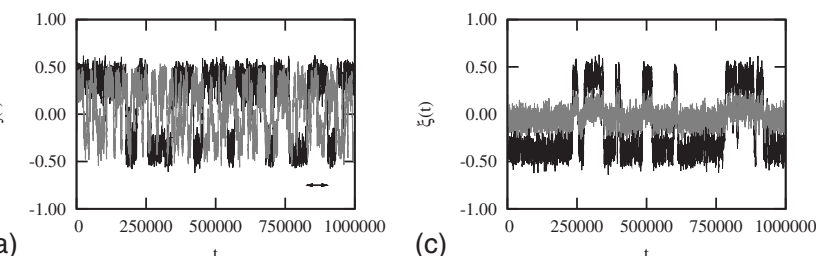

(c)
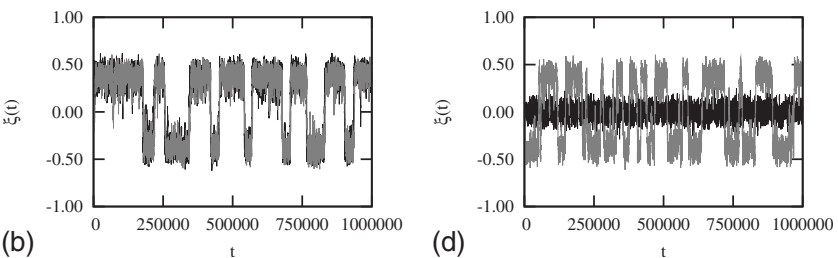

FIG. 9. The results of coupling two networks of stochastic oscillators depend on their coupling constants. Both networks $S$ and $P$ were realized with $N=1000$ clocks and unperturbed transition rate $g=0.01$. The coupling was realized by connecting $F N=20$ nodes of network $S$ with all nodes of network $P$. The perturbation coupling constant was $K_{P S}=2$ in all cases. The fluctuations of the mean field $\xi(t)$ is depicted by gray and black lines for networks $S$ and $P$, respectively. (a) Uncoupled case, $K_{P}=1.06, K_{S}=1.03$. (b) Introducing the coupling between network $P$ and network $S$ leads to the synchronization. $K_{P}=1.06, K_{S}=1.03$. (c) Synchronization is not present when one of the networks is not in the complex regime. Here, perturbed network $S$, with $K_{S}=0.80$, does not respond to the complex perturbation of the network $P$, where $K_{P}=1.06$. (d) Synchronization is also absent in the case when the perturbing network $P$ is not in the complex regime. Here perturbed network $S$, with $K_{S}=1.06$ does not respond to the perturbation of the network $P$, where $K_{P}=0.80$.

$$
\chi=\lim _{t \rightarrow \infty}\left\langle\xi_{P}(t) \xi_{S}(t)\right\rangle
$$

as an average over a very large number of realizations of this perturbation experiment. In the three-dimensional representation of Fig. 10 we illustrate the result of this analysis.

The results of Fig. 10 are impressive and surprising. It is evident that when one cluster is self-organized and the other is not, synchronization is very weak or vanishing. When both clusters are self-organized and characterized by values of $K$

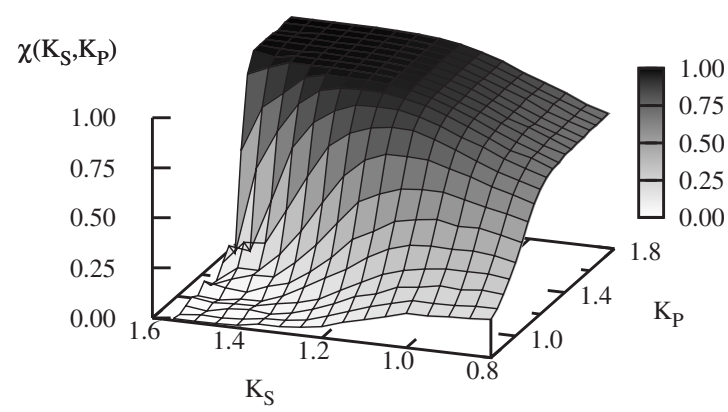

FIG. 10. The cross correlation between the fluctuation of a perturbed network $S$ and perturbing network $P$ as a function of their coupling parameter $K$. The network $P$ perturbs only two nodes $(F N=2)$ of the network $S$ with the coupling $K_{P S}=2$. Both networks have 100 nodes. 
well above the phase-transition threshold, synchronization becomes perfect.

To appreciate the importance of this result, it is convenient to mention the recent work for the extension of the well-known linear response theory (LRT) of Kubo [32] to the case of event-dominated dynamics. The traditional LRT requires the use of stationary correlation functions and cannot be used to study irretrievably nonstationary processes such as our decision-making model. For this reason LRT has been suitably generalized by the authors in Refs. [33-35] while maintaining the rigorously linear dependence on the perturbation strength of the original theory. According to the generalized LRT, the mean value over infinitely many realizations of the response of $S$ to the same perturbation $\xi_{p}(t)$ is given by

$$
\left\langle\xi_{S}(t)\right\rangle=\epsilon \int_{0}^{t} d s\left(\frac{d}{d t} \Psi_{S}(t, s)\right) \xi_{P}(s),
$$

where

$$
\Psi_{S}(t, s) \equiv\left\langle\xi_{S}(t) \xi_{S}(s)\right\rangle
$$

is the nonstationary correlation function of $\xi_{S}(t)$. Note that this LRT requires $\xi_{S}$ to be a renewal non-Poisson process, a condition fulfilled by the fluctuation $\xi_{S}$ of our decisionmaking model, as proved in Sec. IV. The parameter $\epsilon$, defining the perturbation strength, is a real number of the interval $[0,1]$. However, to generate a LRT the authors in Refs. [33-35] had to set the important condition

$$
\epsilon \ll 1 \text {. }
$$

It is easy to prove [36] that Eq. (43) yields

$$
\left\langle\xi_{S}(t) \xi_{S}(s)\right\rangle=\epsilon \int_{0}^{t} d s\left(\frac{d}{d t} \Psi_{S}(t, s)\right) \Psi_{P}(t, s),
$$

where

$$
\Psi_{P}(t, s) \equiv\left\langle\xi_{P}(t) \xi_{P}(s)\right\rangle
$$

is the nonstationary correlation function of $\xi_{P}(t)$. The result of Eq. (46) can be proved in the case when $\xi_{S}$ is a dichotomous renewal process [33-35]. The renewal condition is not necessary for $\xi_{P}(t)$, which, in principle, can also be a regular function of time. In the case when both $\xi_{S}(t)$ and $\xi_{P}(t)$ are Poisson dichotomous noises, with laminar regions whose time durations are derived from the waiting-time distribution densities,

$$
\psi_{S}(\tau)=g_{S} \exp \left(-g_{S} \tau\right)
$$

and

$$
\psi_{P}(\tau)=g_{P} \exp \left(-g_{P} \tau\right)
$$

respectively, it is straightforward to prove [36] that

$$
\underline{\chi}=\frac{g_{S}}{g_{S}+g_{P}} .
$$

According to the CM principle [21] the maximum efficiency of the information transport is reached when the perturbed system shares the complexity of the perturbing sys-

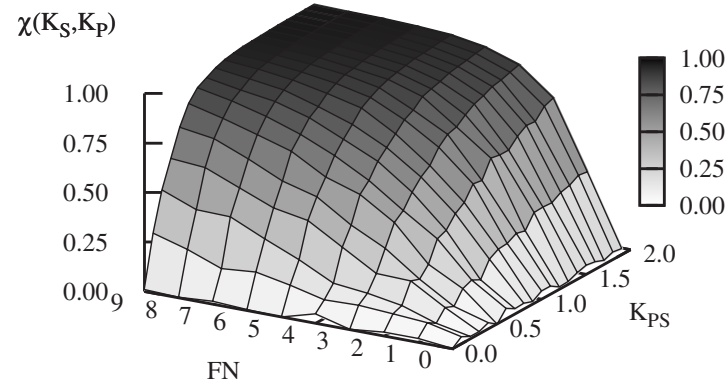

FIG. 11. A three-dimensional graph of the cross-correlation intensity $\chi$. Both system $S$ and system $P$ have 100 nodes. $\chi$ is plotted as a function of the number of intercluster links $F N$ and of the intercluster interaction intensity $K_{P S} . K_{S}=K_{P}=1.3$. The numerical results have been obtained using sequences of length $L=10^{8}$. Although the statistics are not yet accurate enough to define the transition from the region of low or vanishing correlation, it is clear that in the region $0<K_{P S}<1$ and $0<F N<5$, there is small or vanishing correlation. The linear response theory is expected to hold true in this region.

tem. The event-dominated LRT supports the CM principle, insofar as $\chi=\epsilon$ [37], which is the maximum as possible synchronization is compatible with the linear response to a weak perturbation.

After this digression, we can explain why the results of Fig. 10 are surprising. Although the decision-making model of this paper fits the conditions necessary for the eventdominated LRT to work, with only $2 \%$ of the nodes of $S$ perturbed by $P$ and $K_{S} \approx 1.2$ and $K_{P} \approx 1.2, \chi$ turns out to be very close to 1 , thereby forcing us to set $\epsilon=1$, a condition inconsistent with the LRT. This is confirmed by Fig. 9(b) showing that the time length of the laminar regions of $S$ is drastically changed by the $P$ perturbation, whereas the LRT is valid when the dynamical properties of $S$ are virtually indistinguishable from those in the absence of perturbation.

We are not yet in a position to determine analytically the perturbation parameter $\epsilon$ so as to support numerically the theoretical prediction that this decision model must obey the event-dominated LRT. However, the numerical results of Fig. 11 allow us to establish that, if it does, the region of LRT validity is restricted to a portion of the phase space $\left(F N, K_{P S}\right)$.

We see that the correlation indicator $\chi$ vanishes at $F N$ $=0$ and it becomes smaller and smaller if $F N>0$ when $K_{P S}$ becomes smaller and smaller than 1 . Figure 11 shows that in the region approximately corresponding to $K_{P S}>1$ and $F N$ $>5$, we should set $\epsilon=1$ to make the results compatible with the nonstationary LRT. Since we expect that $\chi<1$ is the condition for the validity of the LRT, we must conclude that the LRT may hold true for $K_{P S}<1$ and $F<0.02$. The decision-making model of this paper generates a surprisingly accurate synchronization with a small number of perturbed nodes of $S$, provided that the control parameter $K_{P S}$ is sufficiently large $\left(K_{P S}>1.5\right)$. In the whole phase space of Fig. 11 the system $S$ partially correlates with $P$. This partial correlation becomes an almost exact form of synchronization of $S$ with $P$ in the plateau region $K_{P S}>1$ and $F N>5$.

In conclusion, the decision-making model of this paper generates a significantly large value of $\chi$ with $K_{P}<1$ and 


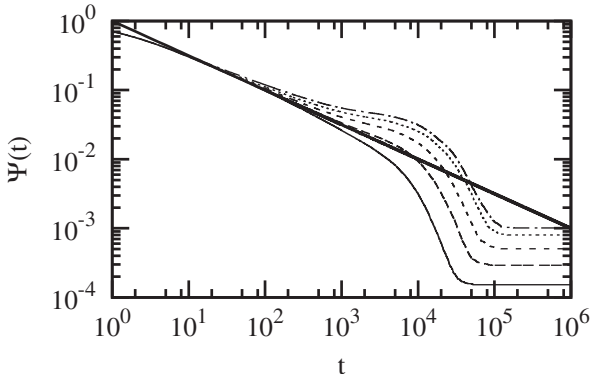

FIG. 12. Survival probability function $\Psi(t)$ for a system of four coupled clusters as a function of coupling strength. Each cluster consisted of $N=500$ clocks with the coupling constant $K=1.05$ and $g=0.01$. The coupling was realized by connecting $n$ nodes from each cluster with other clusters. Thin solid line for $n=1$, long dashed line for $n=5$, short dashed line for $n=10$, dotted line for $n$ $=20$, and dashed dotted line for $n=50$ common nodes.

$F<0.02$ and a fast increase in $\chi$ to the maximum value of 1 with a modest increase in $K_{P}$ and $F$. We note that the CM phenomenon has been conjectured on the basis of a generalization of the well-known LRT of Kubo [32], which allows the perturbed system to maintain its unperturbed dynamic properties. The decision-making model of this paper indicates that the CM principle is not limited to the LRT condition: it can generate exact synchronization, with the perturbed system forced to adopt the dynamical properties of the perturbing system in spite of the fact that $P$ perturbs a surprisingly small fraction of $S$ nodes.

\section{PARTITIONING THE NETWORK}

As pointed out in Sec. II C, the adoption of the mean-field approach yielding Eq. (17) is legitimate only when a single all-to-all coupling cluster is considered. When the network consists of several clusters weakly coupled together the condition for the adoption of Eq. (17) is violated. In Fig. 12 we show the effect of partitioning the network into four interacting parts. We see that a critical value of the coupling among the four cluster exists $(K=5)$ which has the effect of recovering the Kramers shoulder as an effect of the cooperation among clusters (in this case the coupling among clusters is bidirectional) without using the all-to-all coupling condition. The long dashed line of Fig. 12, corresponding to intercluster interaction with five nodes indicates that the inverse powerlaw region is not confined to $t<\tau_{S}$. We believe that in a very large network, not fulfilling the all-to-all condition, the very fat Kramers shoulder may be converted into a significant extension of the inverse power law showing up in the Suzuki time region.

\section{CONCLUSIONS AND PLANS FOR FUTURE RESEARCH WORK}

In this paper we show that the decision-making model in Refs. [14-16] can be used to discuss the consensus emer- gence with theoretical arguments resting on the dynamic bistability of Kramers theory [20]. It is surprising that the Kramers theory 68 years after its publication is still the subject of research and debate. The reason is that the dynamic bistability is ubiquitous and acts under conditions different from the idealized condition originally set by Kramers. In some cases [18] the quantum and classical noise plays dual role. We note that quantum noise was ignored in the original work of Kramers [20]. In other cases [19] the action of nonGaussian noise rather than the white noise of Kramers is considered. Finally, the new condition of a potential barriers of very small intensity [26] can be considered. This makes it possible to reveal the existence of an extended time region departing from the Poisson condition of Kramers theory.

A very important result of the present paper is in fact the discovery that the Kramers exponential tail is a source of complexity. In the temporally wide region between $\tau_{S}$ and $\tau_{K}$, in fact, the survival probability $\Psi(t)$ is much higher than the $\Psi(t)$ of complex networks whose inverse power-law behavior with $\mu=1.5$ was truncated at a time $t_{\text {trunc }}$ on the order of $\tau_{K}$. In a sense, the ergodicity breakdown is even more intense than the ergodicity breakdown realized with complex networks characterized by the ordinary upper truncation [30].

In conclusion, the extended Suzuki time region is responsible for a striking departure from the Poisson condition of Kramers theory. The CM phenomenon is a clear sign of the breakdown of this departure. The conventional Poisson condition would generate only a modest synchronization between two different networks [36]. We show, on the contrary, that with our decision-making model the CM effects reach the condition of maximal efficiency.

We believe that this property will have important applications in the wide field of decision-making processes. A complex network can be interpreted as a set of interacting clusters which exchange information so as to turn the local into global consensus. This paper shows that this transfer of information is realized through the crucial role of renewal nonPoisson events. According to [21] the efficient transport of information occurs in the nonergodic regime, and herein we have confirmed this theoretical expectation. On the basis of this property it will be possible to address the study of communication transport through networks of any kind regardless of size. This will have the effect of establishing a connection between structural and dynamic complexities.

\section{ACKNOWLEDGMENT}

We gratefully acknowledge the financial support of the U.S. Army Research Office (Grant No. W911NF-08-1-0177). 
[1] L. Conradt and C. List, Philos. Trans. R. Soc. London, Ser. B 364, 719 (2009).

[2] J. R. G. Dyer, A. Johansson, D. Helbing, I. D. Couzin, and J. Krause, Philos. Trans. R. Soc. London, Ser. B 364, 781 (2009).

[3] D. J. T. Sumpter and S. C. Pratt, Philos. Trans. R. Soc. London, Ser. B 364, 743 (2009).

[4] J.-P. Eckmann, O. Feinerman, L. Gruendlinger, E. Moses, J. Soriano, and T. Tlusty, Phys. Rep. 449, 54 (2007).

[5] O. Feinerman and E. Moses, J. Neurosci. 26, 4526 (2006).

[6] D. J. Watts and S. H. Strogatz, Nature (London) 393, 440 (1998).

[7] A. L. Barabási and R. Albert, Science 286, 509 (1999).

[8] P. Holme and B. J. Kim, Phys. Rev. E 65, 026107 (2002).

[9] J. Wang and L. Rong, in Proceedings of the 2008 International Conference on Computer Science and Information Technology, ICCSIT '08, Singapore, 2008 (IEEE, New York, 2008), pp. 143-146.

[10] N. Päivinen, Pattern Recogn. Lett. 26, 921 (2005).

[11] V. Latora and M. Marchiori, Phys. Rev. Lett. 87, 198701 (2001).

[12] A. Grönlund, P. Holme, and P. Minnhagen, EPL 81, 28003 (2008).

[13] A. Arenas, A. Díaz-Guilera, J. Kurths, Y. Moreno, and C. Zhou, Phys. Rep. 469, 93 (2008).

[14] K. Wood, C. Van den Broeck, R. Kawai, and K. Lindenberg, Phys. Rev. Lett. 96, 145701 (2006).

[15] T. Prager, B. Naundorf, and L. Schimansky-Geier, Physica A 325, 176 (2003)

[16] S. Bianco, E. Geneston, P. Grigolini, and M. Ignaccolo, Physica A 387, 1387 (2008).

[17] S. Bianco, P. Grigolini, and P. Paradisi, J. Chem. Phys. 123, 174704 (2005).

[18] M. I. Dykman, Applications of Nonlinear Dynamics, Understanding Complex Systems(Springer, Berlin, 2009), p. 367.
[19] L. Billings, M. I. Dykman, and I. B. Schwartz, Phys. Rev. E 78, 051122 (2008).

[20] H. A. Kramers, Physica (Amsterdam) 7, 284 (1940).

[21] B. J. West, E. L. Geneston, and P. Grigolini, Phys. Rep. 468, 1 (2008).

[22] R. Failla, M. Ignaccolo, P. Grigolini, and A. Schwettmann, Phys. Rev. E 70, 010101(R) (2004).

[23] M. Suzuki, Phys. Lett. A 67, 339 (1978).

[24] P. Allegrini, F. Barbi, P. Grigolini, and P. Paradisi, Phys. Rev. E 73, 046136 (2006).

[25] A. Rebenshtok and E. Barkai, J. Stat. Phys. 133, 565 (2008).

[26] G. Margolin and E. Barkai, Phys. Rev. E 72, 025101(R) (2005).

[27] X. Brokmann, J.-P. Hermier, G. Messin, P. Desbiolles, J.-P. Bouchaud, and M. Dahan, Phys. Rev. Lett. 90, 120601 (2003).

[28] G. Margolin, V. Protasenko, M. Kuno, and M. Barkai, Adv. Chem. Phys. 133, 327 (2006), special edition.

[29] G. Margolin, V. Protasenko, M. Kuno, and M. Barkai, J. Phys. Chem. B 110, 19053 (2006).

[30] I. Chung and M. G. Bawendi, Phys. Rev. B 70, 165304 (2004); Y. Nishiyama, Phys. Rev. E 75, 011106 (2007).

[31] I. Chung, J. B. Witkoskie, J. P. Zimmer, J. Cao, and M. G. Bawendi, Phys. Rev. B 75, 045311 (2007).

[32] R. Kubo, Can. J. Phys. 34, 1274 (1956).

[33] F. Barbi, M. Bologna, and P. Grigolini, Phys. Rev. Lett. 95, 220601 (2005).

[34] P. Allegrini, M. Bologna, P. Grigolini, and B. J. West, Phys. Rev. Lett. 99, 010603 (2007).

[35] G. Aquino, P. Grigolini, and B. J. West, EPL 80, 10002 (2007).

[36] M. Luković, M. Ignaccolo, L. Fronzoni, and P. Grigolini, Phys. Lett. A 372, 2608 (2008).

[37] G. Aquino, M. Bologna, P. Grigolini, and B. J. West (unpublished). 